15

\title{
Новые возможности позитронно-эмиссионной томографии
}

\author{
(C) А.Н. Волобуев \\ Самарский государственный медицинский университет, \\ Кафедра медицинской и биологической физики, \\ 443099 Самара, Россия \\ e-mail: volobuev47@yandex.ru
}

(Поступило в Редакцию 25 января 2017 г.)

Исследованы причины возникновения углового распределения фотонов, образующихся при аннигиляции электрона и позитрона в позитронно-эмиссионном томографе. Показано, что угловое распределение интенсивности излучения, т.е. вероятности излучения фотонов по различным углам, является следствием эффекта Доплера в системе отсчета центра масс электрона и позитрона. В системе отсчета, связанной с электроном, угловое распределение количества вылетающих фотонов отсутствует, но заменяется доплеровским изменением частот фотонов. Полученные результаты позволяют расширить возможности позитронно-эмиссионного томографа при диагностике заболеваний и дополнительно получить механические характеристики ткани организма: плотность, вязкость и т.д.

DOI: 10.21883/JTF.2018.01.45498.2178

\section{Введение}

Позитронно-эмиссионная томография - это метод радиоизотопной диагностики, в котором используется внутривенное введение радиофармацевтических препаратов (РФП), излучающих позитроны [1]. Позитроны возникают в результате $\beta$-позитронного распада короткоживущих радиофармацевтических изотопов, например $C_{6}^{11}, O_{8}^{15}, N_{7}^{13}, F_{9}^{18}, P_{15}^{30}$. Поэтому важным узлом позитронно-эмиссионного томографа (ПЭТ) является циклотрон, вырабатывающий эти короткоживущие радиоизотопы с периодом полураспада $T_{1 / 2} \sim 10-20 \mathrm{~min}$. Испущенные ядром изотопа позитроны $e_{+1}^{0}$, сталкиваясь с электронами $e_{-1}^{0}$, которых всегда много в веществе, аннигилируют $e_{-1}^{0}+e_{+1}^{0} \rightarrow 2 \hbar \omega(\hbar-$ постоянная Планка, $\omega$ - циклическая частота фотона). В результате появляется два $\gamma$-кванта (фотона) (рис. 1).

Особенностью этих $\gamma$-квантов, с энергией $\sim 511 \mathrm{keV}$ является то, что они излучаются практически в строго противоположных направлениях.

В ПЭТ кушетка с пациентом, у которого в тканях имеется патологический участок 2 , в котором накопился РФП, помещается в кольцо 1, состоящее из детекторов, регистрирующих $\gamma$-кванты. Информационную ценность имеет только событие, когда два излученных в процессе аннигиляции $\gamma$-кванта достигают противоположных детекторов одновременно, т.е. оба $\gamma$-кванта пролетают одинаковый путь до детекторов. Например, $\gamma$-кванты, излученные из точки $a$ патологического очага, достигают двух детекторов $\gamma$-излучения $D_{1}$ и $D_{2}$, расположенных на линии $A-A$, одновременно, эти сигналы участвуют в построении изображения.

Одновременность регистрации $\gamma$-квантов служит признаком того, что ЭВМ, на которую поступают сигналы от детекторов, должна использовать полученные сигналы для формирования изображения.
Координата, излучающей $\gamma$-кванты точки патологического очага находится на линии, соединяющей детекторы точно посередине между ними. Интенсивность сигнала от детекторов указывает на количество накопленного РФП.

Сигналы детекторов, не одновременно регистрирующих $\gamma$-кванты, игнорируются. Это связано с тем, что для таких $\gamma$-квантов невозможно рассчитать точку их испускания в связи с очень большой скоростью движения $\gamma$-квантов.

При перемещении кушетки с пациентом вдоль оси, перпендикулярной плоскости кольца детекторов, получается трехмерное $(3 D)$ изображение распределения концентрации РФП в патологическом очаге.

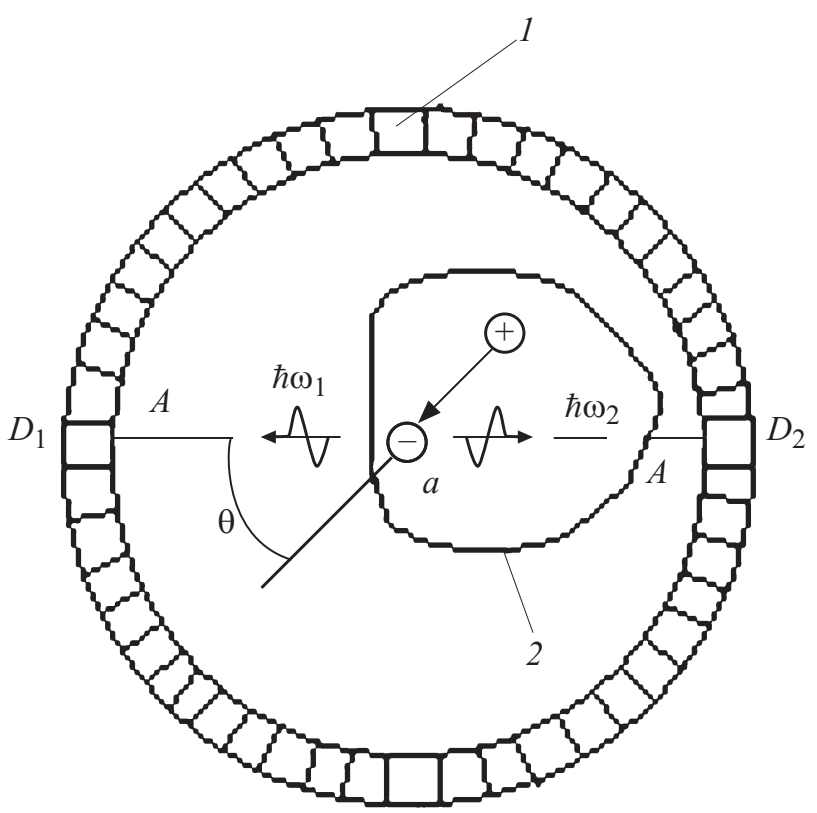

Рис. 1. Схема регистрации фотонов в ПЭТ. 
Результаты регистрации $\gamma$-квантов всеми одновременно срабатывающими детекторами, обрабатываются с помощью ЭВМ. При этом получается изображение высокой информативности.

Разрешающая способность ПЭТ определяется в основном длиной пробега позитрона до аннигиляции и составляет 4-6 mm. Метод позитронно-эмиссионной томографии в настоящее время является наиболее информативным по сравнению с другими диагностическими методами, включая компьютерную рентгеновскую томографию и магниторезонансную томографию. Он успешно применяется прежде всего для раннего обнаружения онкологических новообразований и особенно метастазов. С его помощью обнаруживаются патологические очаги, когда структурных изменений еще нет, но течение биохимических реакций в клетках уже нарушено.

Широкое использование ПЭТ сдерживается его исключительно высокой стоимостью, однако, по нашему мнению, возможности ПЭТ используются далеко не полностью.

Целью настоящей работы является показать, как с помощью ПЭТ можно найти некоторые механические характеристики тканей организма: плотность, вязкость и т.д.

\section{Угловое и энергетическое распределения аннигиляционного излучения}

Для этого проанализируем угловое распределение вылетающих фотонов энергией $\hbar \omega$ при аннигиляции позитрона и электрона в ПЭТ. К сожалению, механизм процесса аннигиляции электрона и позитрона до настоящего времени неизвестен. П. Дираком была предложена модель этого процесса.

Согласно представлениям Дирака [2,3], аннигиляцию можно представить как переход электрона из состояния с положительной энергией в состояние с отрицательной энергией. Согласно теории вакуумных дырок Дирака, позитрон представляет собой дырку в поле вакуума. Взаимодействие электрона и позитрона, их аннигиляция - это заполнение вакуумной дырки электроном. При этом выделяется энергия в виде двух квантов электромагнитного излучения.

Квантово-электродинамические расчеты процесса аннигиляции были проведены довольно давно, неоднократно проверялись и перепроверялись.

В результате этих расчетов были получены две формулы для дифференциального эффективного сечения рассеяния квантов электромагнитного излучения в телесный угол $d \Omega$.

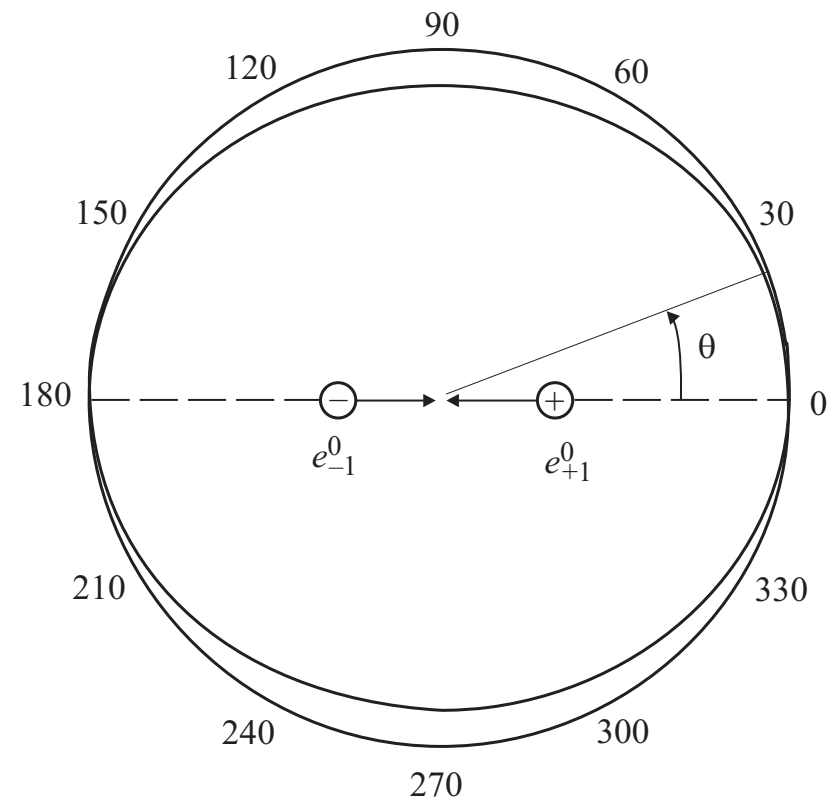

Рис. 2. Угловое распределение аннигиляционного излучения.

Первая по времени формула была получена Гайтлером [4]. Эта формула имеет вид

$$
\begin{aligned}
d \sigma= & \frac{e^{4}}{4(4 \pi)^{2} k^{0} p}\left\{\frac{\left(k^{0}\right)^{2}+p^{2}+p^{2} \sin ^{2} \theta}{\left(k^{0}\right)^{2}-p^{2} \cos ^{2} \theta}\right. \\
& \left.-\frac{2 p^{4} \sin ^{4} \theta}{\left(\left(k^{0}\right)^{2}-p^{2} \cos ^{2} \theta\right)^{2}}\right\} d \Omega .
\end{aligned}
$$

Формула дана в обозначениях [5], где имеется ее подробный вывод. Использована так называемая рациональная система единиц, в которой скорость света и постоянная Планка равны единице $c=\hbar=1$. В этой системе единиц энергия импульс и масса имеют одну и ту же размерность.

В формуле (1) $e$ - заряд электрона (или позитрона с противоположным знаком), $k^{0}$ - энергия фотона, $p$ - импульс электрона, $\theta-$ угол между импульсами электрона и одного из излученных фотонов. Формула (1) получена при условии суммирования по всем направлениям поляризации фотонов.

При выводе (1) использована система отсчета, связанная с центром масс взаимодействующих электрона и позитрона, в которой импульсы электрона и позитрона равны по модулю между собой и противоположны по направлению $p_{1}=-p_{2}=p$. Импульсы фотонов также равны по модулю между собой и противоположны по направлению $k_{1}=k_{2}[4,5]$. Отметим, что в этой системе отсчета условия наблюдения обоих фотонов одинаковы.

На рис. 2 показано угловое распределение $\frac{d \sigma}{d \Omega}=f(\theta)$ согласно формуле (1). Это распределение показано для отношения $\frac{p^{2}}{\left(k^{0}\right)^{2}}=0.2$. Коэффициент перед квадратными скобками в (1) принят равным единице. График на рис. 2 
имеет иллюстративный характер, так как будет показано ниже,

$$
\frac{p^{2}}{\left(k^{0}\right)^{2}}=\frac{\alpha^{2}}{4} \sim 10^{-5}
$$

где $\alpha \approx \frac{1}{137}$ постоянная тонкой структуры. В этом случае распределение $\frac{d \sigma}{d \Omega}$ практически сферически симметрично.

Вторая формула была предложена несколько позже Фейнманом [6]. Она представляет собой частотное или энергетическое распределение вылетающих квантов

$$
\begin{aligned}
d \sigma= & \frac{e^{4} \omega_{1}^{2}}{64 \pi^{2} 4 m^{2}\left|\mathbf{p}_{+}\right|\left(E_{+}+m\right)} \\
& \times\left[\frac{\omega_{1}}{\omega_{2}}+\frac{\omega_{2}}{\omega_{1}}+2-4\left(\mathbf{e}_{1} \mathbf{e}_{2}\right)^{2}\right] d \Omega .
\end{aligned}
$$

Формула (2) записана в обозначениях [6]. Как и в предыдущем случае, использована рациональная система единиц.

В формуле (2) $\mathbf{e}_{1}$ и $\mathbf{e}_{2}$ - единичные векторы поляризации излученных при аннигиляции фотонов, $\omega_{1}$ и $\omega_{2}-$ частоты излученных фотонов, $m$ - масса электрона (или позитрона), $\left|\mathbf{p}_{+}\right|-$модуль импульса позитрона, $E_{+}$- его энергия.

Формула (2) похожа на формулу Клейна-Нишины для эффекта Комптона [7]. Главное отличие в том, что перед третьим и четвертым слагаемыми в скобках знаки изменены на противоположные.

Важнейшим отличительным условием вывода формулы (2) является использование другой системы отсчета по сравнению с выводом формулы (1). Формула (2) выводилась в системе отсчета, в которой электрон покоится, а движется позитрон.

Эта система отсчета в целом эквивалентна системе отсчета, связанной с ПЭТ. Поэтому назовем эту систему отсчета лабораторной. Электроны объекта, исследуемого в ПЭТ, в основном находятся в связанном состоянии. Поэтому электроны в лабораторной системе отсчета можно считать неподвижными (если исключить хаотическое тепловое движение молекул).

Обе формулы (1) и (2) выводились с помощью стандартной диаграммной техники Фейнмана и диаграмм второго порядка теории возмущений. Однако результаты вывода существенно различаются.

Во-первых, формула (1) предполагает довольно сложное угловое распределение интенсивности $I$ аннигиляционного излучения, так как $d \sigma \sim d P \sim I d \Omega$, где $d P-$ поток энергии излучения через площадь $d S$, интенсивность $I=\frac{d P}{d S}$. Причем это распределение связано только с импульсом электрона, угол $\theta$ присутствует только в комплексе с импульсом $p$. В формуле (2) угловое распределение фотонов в явном виде отсутствует.

Во-вторых, формула (2) предполагает возможность различной энергии фотонов при аннигиляции, что запрещается выводом формулы (1), вследствие $k_{1}=-k_{2}$.
Поэтому прежде всего возникает вопрос, какова природа углового распределения интенсивности аннигиляционного излучения в (1)? Связано ли это распределение с самим процессом аннигиляции, т.е. переходом „вещество-энергия“, либо определяется какимито другими эффектами? Сохранится ли данное угловое распределение фотонов при переходе к другой системе отсчета, например, связанной с ПЭТ?

\section{Причины углового и энергетического распределения аннигиляционного излучения}

Для исследования причины угловой зависимости дифференциального эффективного сечения (1) рассмотрим промежуточное выражение вывода, которое еще не просуммировано по направлениям поляризации фотонов [5]

$$
d \sigma=\frac{1}{128 \pi^{2}} \frac{e^{4}}{p k^{0}}\left\{\begin{array}{c}
\frac{\left(k^{0}\right)^{4}}{\left(p k_{1}\right)\left(p k_{2}\right)}-\frac{4\left(k^{0}\right)^{4}\left(p e_{1}\right)^{2}\left(p e_{2}\right)^{2}}{\left(p k_{1}\right)^{2}\left(p k_{2}\right)^{2}}- \\
-\frac{4\left(k^{0}\right)^{2}\left(e_{1} e_{2}\right)\left(p e_{1}\right)\left(p e_{2}\right)}{\left(p k_{1}\right)\left(p k_{2}\right)}-\left(e_{1} e_{2}\right)^{2}
\end{array}\right\} d \Omega,
$$

где $k_{1}$ и $k_{2}-$ импульсы фотонов. Переменные в квадратных скобках: импульс электрона, импульсы фотонов, единичные векторы поляризации фотонов записаны в виде 4 векторов.

Формулу (3) несложно преобразовать к виду

$$
\begin{aligned}
d \sigma= & \frac{1}{128 \pi^{2}} \frac{e^{4}}{p k^{0}}\left\{\frac{\left(k^{0}\right)^{4}}{\left(p k_{1}\right)\left(p k_{2}\right)}\right. \\
& \left.-\left[2\left(k^{0}\right)^{2} \frac{\left(p e_{1}\right)\left(p e_{2}\right)}{\left(p k_{1}\right)\left(p k_{2}\right)}+\left(e_{1} e_{2}\right)\right]^{2}\right\} d \Omega .
\end{aligned}
$$

Перейдем в (4) к пространственным векторам, используя правило $\left.(a b)=a^{0} b^{0}-\mathbf{a b}\right)$, где $\mathbf{a}$ и $\mathbf{b}-$ трехмерные векторы, $a^{0}$ и $b^{0}$ - энергетические компоненты 4-векторов.

Переходя к трехмерным векторам, а также учитывая отсутствие энергетических компонент у поляризационных 4-векторов $e^{0}=0$, выражение (4) можно представить в виде

$$
\begin{aligned}
d \sigma & =\frac{1}{128 \pi^{2}} \frac{e^{4}}{p k^{0}}\left\{\frac{\left(k^{0}\right)^{4}}{\left(k^{0}\right)^{4}-\left(\mathbf{p k}_{1}\right)^{2}}-\left[2\left(k^{0}\right)^{2} \frac{\left(\mathbf{p e}_{1}\right)\left(\mathbf{p e}_{2}\right)}{\left(k^{0}\right)^{4}\left(\mathbf{p k}_{1}\right)^{2}}\right.\right. \\
& \left.\left.+\left(\mathbf{e}_{1} \mathbf{e}_{2}\right)\right]^{2}\right\} d \Omega=\frac{1}{128 \pi^{2}} \frac{e^{4}}{p k^{0}}\left\{\frac{1}{1-\left(\frac{\mathbf{p k}_{1}}{\left(k^{0}\right)^{2}}\right)^{2}}\right. \\
& \left.-\left[\frac{2}{\left(k^{0}\right)^{2}} \frac{\left(\mathbf{p e}_{1}\right)\left(\mathbf{p e}_{2}\right)}{1-\left(\frac{\mathbf{p k}_{1}}{\left(k^{0}\right)^{2}}\right)^{2}}+\left(\mathbf{e}_{1} \mathbf{e}_{2}\right)\right]^{2}\right\} d \Omega .
\end{aligned}
$$

При выводе (5) также использовано условие вылета фотонов в строго противоположных направлениях $k_{2}=-k_{1}$. 


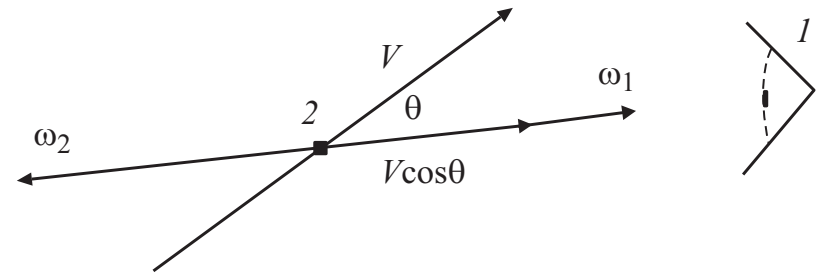

Рис. 3. Наблюдение фотона, который излучается движущейся частицей.

Учитывая $\left|\mathbf{k}_{1}\right|=k^{0}$, а также в соответствии с законом сохранения энергии $c k^{0}=m c^{2}$ (для наглядности вводим внутри скобок скорость света $c=1$ ), в формуле (5) заменим $\frac{\mathbf{p k}_{1}}{\left(k^{0}\right)^{2}}=\frac{V}{c} \cos \theta$, где $V-$ скорость электрона. В результате получим

$$
\begin{aligned}
d \sigma & =\frac{1}{128 \pi^{2}} \frac{e^{4}}{p k^{0}}\left\{\frac{1}{1-\left(\frac{V}{c} \cos \theta\right)^{2}}\right. \\
& \left.-\left[\frac{2}{1-\left(\frac{V}{c} \cos \theta\right)^{2}} \frac{\left(\mathbf{p e}_{1}\right)\left(\mathbf{p e}_{2}\right)}{\left(k^{0}\right)^{2}}+\left(\mathbf{e}_{1} \mathbf{e}_{2}\right)\right]^{2}\right\} d \Omega .
\end{aligned}
$$

Перейдем в (6) в лабораторную систему отсчета, связанную с электроном. В этом случае $p=0$, a $V-$ можно рассматривать как скорость движения позитрона. То же самое относится и к величине $p$ в коэффициенте перед скобками. В данной системе отсчета формула (6) значительно упрощается

$$
d \sigma=\frac{1}{128 \pi^{2}} \frac{e^{4}}{p k^{0}}\left\{\frac{1}{1-\left(\frac{V}{c} \cos \theta\right)^{2}}-\left(\mathbf{e}_{1} \mathbf{e}_{2}\right)^{2}\right\} d \Omega .
$$

Для дальнейшего анализа исследуем вспомогательную задачу.

Наблюдатель 1, находящийся в „неподвижной“ (связанной с Землей) системе отсчета (рис. 3), рассматривает некоторую движущуюся со скоростью $V$ частицу 2 , которая в определенный момент времени излучает два противоположно направленных кванта. При $V=0$ частоты квантов равны $\omega_{0}$. Угол между скоростью частицы и направлением распространения одного из квантов равен $\theta$. В направлении наблюдателя частица имеет составляющую скорости $V \cos \theta$.

За счет эффекта Доплера квант, движущийся в направлении наблюдателя, будет обладать повышенной частотой [8]

$$
\omega_{1}=\omega_{0} \frac{\sqrt{1-\frac{V^{2}}{c^{2}}}}{1-\frac{V}{c} \cos \theta} .
$$

Для кванта, движущегося в противоположном направлении, будет наблюдаться так называемое „красное смещение

$$
\omega_{2}=\omega_{0} \frac{\sqrt{1-\frac{V^{2}}{c^{2}}}}{1+\frac{V}{c} \cos \theta} .
$$

Используя (8) и (9), найдем величину комплекса $\frac{\omega_{1}}{\omega_{2}}+\frac{\omega_{2}}{\omega_{1}}+2$, который входит в формулу $(2)$ :

$$
\frac{\omega_{1}}{\omega_{2}}+\frac{\omega_{2}}{\omega_{1}}+2=\frac{\left(\omega_{1}+\omega_{2}\right)^{2}}{\omega_{1} \omega_{2}}=\frac{4}{1-\left(\frac{V}{c} \cos \theta\right)^{2}} .
$$

Отметим, что различие в частотах квантов в рассматриваемой задаче определяется различием в условиях наблюдения этих квантов: один квант движется к наблюдателю, другой удаляется от него.

В формуле (7) фактически реализуется рассмотренная вспомогательная задача. При этом под движущейся частицей подразумевается позитрон, а наблюдатель находится на „неподвижном“ электроне. Поэтому, подставляя (10) в (7), найдем

$$
\begin{aligned}
d \sigma & =\frac{1}{128 \pi^{2}} \frac{e^{4}}{p k^{0}}\left\{\frac{1}{4}\left(\frac{\omega_{1}}{\omega_{2}}+\frac{\omega_{2}}{\omega_{1}}+2\right)-\left(\mathbf{e}_{1} \mathbf{e}_{2}\right)^{2}\right\} d \Omega \\
& =\frac{1}{128 \pi^{2}} \frac{e^{4}}{4 p k^{0}}\left\{\frac{\omega_{1}}{\omega_{2}}+\frac{\omega_{2}}{\omega_{1}}+2-4\left(\mathbf{e}_{1} \mathbf{e}_{2}\right)^{2}\right\} d \Omega
\end{aligned}
$$

При использовании формулы (10) мы фактически отказались от условия $k_{2}=-k_{1}$.

Если в коэффициенте перед скобками в формуле (2) принять $k^{0}=E_{+}=m=\omega_{1}$, то формулы (2) и (11) становятся тождественными.

Отметим еще один существенный момент, проявляющийся при переходе от системы отсчета, связанной с центром масс электрона и позитрона, к лабораторной системе отсчета, связанной с электроном. Если поделить формулу (9) на формулу (8), то получается результат, отличающийся от результата, полученного в монографиях, например, [9,10].

При делении (9) на (8) и принимая $c=1$, получаем

$$
\frac{\omega_{2}}{\omega_{1}}=\frac{k_{2}}{k_{1}}=\frac{1-V \cos \theta}{1+V \cos \theta} .
$$

В $[9,10]$ предлагается следующее соотношение:

$$
\frac{k_{2}}{k_{1}}=\frac{E_{+}-p_{+} \cos \theta}{m} .
$$

Учитывая $E_{+}=m$ и $p_{+}=m V$, находим

$$
\frac{k_{2}}{k_{1}}=1-V \cos \theta \text {. }
$$

Формула (14) несколько отличается от формулы (12). Это связано с тем, что формула (14) получена в рамках первого приближения теории возмущений. Поэтому она принципиально неточная. Формула (12) следует из точных формул эффекта Доплера. Таким образом, оставаясь только в рамках первого приближения теории возмущений, невозможно установить эквивалентность формул (1) и (2). 
В заключение просуммируем формулу (11) по поляризациям фотонов. Возвращаясь к поляризационным 4 векторам с учетом $e^{0}=0$ и используя $\sum_{e_{1} e_{2}}\left(e_{1} e_{2}\right)^{2}=2[5]$, найдем

$$
\begin{aligned}
d \sigma & =\frac{1}{128 \pi^{2}} \frac{e^{4}}{4 p k^{0}}\left\{\left|\frac{\omega_{1}}{\omega_{2}}+\frac{\omega_{2}}{\omega_{1}}+2-4\left(e_{1} e_{2}\right)^{2}\right|\right\} d \Omega \\
& =\frac{1}{128 \pi^{2}} \frac{e^{4}}{p k^{0}}\left\{\left|1-\frac{\left(\omega_{2}-\omega_{1}\right)^{2}}{4 \omega_{1} \omega_{2}}\right|\right\} d \Omega .
\end{aligned}
$$

Знак модуля принят вследствие стандартного использования модуля составного матричного элемента при нахождении дифференциального эффективного сечения процесса [4].

\section{Применение аннигиляционного излучения в ПЭТ}

Учитывая, что в ПЭТ скорости позитронов невелики, а также учитывая (8) и (9), можно записать

$$
\omega_{1} \omega_{2}=\omega_{0}^{2} \frac{1-\frac{V^{2}}{c^{2}}}{1-\left(\frac{V}{c}\right)^{2} \cos ^{2} \theta} \approx \omega_{0}^{2} .
$$

Подставляя (16) в (15), получим

$$
d \sigma \approx \frac{1}{128 \pi^{2}} \frac{e^{4}}{p k^{0}}\left\{\left|1-\frac{(\Delta \omega)^{2}}{4 \omega_{0}^{2}}\right|\right\} d \Omega,
$$

где обозначено $\Delta \omega=\omega_{2}-\omega_{1}$.

Найдем разность частот излучаемых фотонов, т.е. величину $\Delta \omega=\omega_{1}-\omega_{2}$, используя формулы (8) и (9):

$$
\begin{aligned}
\Delta \omega & =\omega_{0} \frac{\sqrt{1-\frac{V^{2}}{c^{2}}}}{1-\frac{V}{c} \cos \theta}-\omega_{0} \frac{\sqrt{1-\frac{V^{2}}{c^{2}}}}{1+\frac{V}{c} \cos \theta} \\
& =\omega_{0} \frac{2 \frac{V}{c} \cos \theta \sqrt{1-\frac{V^{2}}{c^{2}}}}{1-\left(\frac{V}{c} \cos \theta\right)^{2}} .
\end{aligned}
$$

При аннигиляции позитрона и электрона, находящегося в точке $a$ ПЭТ (рис. 1), вылетают два $\gamma$-кванта с энергиями $\hbar \omega_{1}$ и $\hbar \omega_{2}$ в противоположных направлениях. Детекторы в кольце 1 с точки зрения эффекта Доплера в системе отсчета, связанной с электронами, играют роль неподвижных наблюдателей.

По количеству квантов, вылетающих в разных направлениях, процесс сферически симметричен. Поэтому плотность детекторов в кольце 1 должна быть равномерной. Однако частоты квантов, а следовательно, и их энергии, в зависимости от направления на детектор $\theta$ за счет эффекта Доплера могут различаться на величину $\Delta \omega=\omega_{2}-\omega_{1}$.

Если угол $\theta=0$, т.е. позитрон движется по линии $A-A$, соединяющей детекторы $\gamma$-излучения $D_{1}$ и $D_{2}$, разность частот фотонов будет максимально возможной и формула (18) преобразуется к виду

$$
\Delta \omega_{\max }=\omega_{0} \frac{2 \frac{V}{c}}{\sqrt{1-\frac{V^{2}}{c^{2}}}} .
$$

Учитывая $V \ll c$, найдем

$$
\frac{\Delta \omega_{\max }}{2 \omega_{0}}=\frac{V}{c} .
$$

Величину $\omega_{0}$ можно получить, исходя из приближенного равенства $\hbar \omega_{0} \approx m c^{2}$. В этом случае

$$
\Delta \omega_{\max }=2 \frac{V}{\hbar}
$$

где $\hbar=\frac{\hbar}{m c}=3.86159 \cdot 10^{-13} m-$ комптоновская длина волны электрона [11].

Оценим величину $\Delta \omega_{\max }$. Позитрон при распаде используемого в ПЭТ радиофармацевтического элемента вылетает с очень большой скоростью, близкой к скорости света. Но двухфотонная аннигиляция возможна только при малой скорости частиц через образование позитрония [7]. При большой энергии позитрона возникают либо мезоны, либо адроны. Поэтому для возникновения двухфотонной аннигиляции позитрон должен значительно замедлиться. Энергия позитрония равна

$$
E=m c^{2} \frac{\alpha^{2}}{4}
$$

где $\alpha \approx \frac{1}{137}-$ постоянная тонкой структуры [7]. Полагая энергию позитрона в электрическом поле электрона $E_{p}=\frac{p^{2}}{2 m}$ равной половине энергии позитрония, находим импульс позитрона из соотношения $m c^{2} \frac{\alpha^{2}}{8}=\frac{p^{2}}{2 m}$. Так как скорость позитрона невелика, $p=m_{0} c \frac{\alpha}{2}$, а скорость позитрона $V=c \frac{\alpha}{2}$. Используя (21), найдем $\Delta \omega_{\max }=\frac{c \alpha}{\lambda} \approx 0.0057 \cdot 10^{21} \mathrm{~s}^{-1}$. Найденная разность частот эквивалентна $3.75 \mathrm{keV}$. Если принять энергию аннигиляционного фотона $511 \mathrm{keV}$, то полученная разность частот составляет $0.73 \%$. Данная величина вполне приемлема для измерения современными детекторами $\gamma$-излучения.

Ранее при оценке реальной формы распределения аннигиляционного излучения (рис. 2) отмечалось, что отношение $\frac{p^{2}}{\left(k^{0}\right)^{2}}=\frac{\alpha^{2}}{4} \sim 10^{-5}$. Действительно, из формулы $m c^{2} \frac{\alpha^{2}}{8}=\frac{p^{2}}{2 m}$ следует

$$
\frac{p^{2} c^{2}}{m^{2} c^{4}}=\frac{p^{2}}{\left(k^{0}\right)^{2}}=\frac{\alpha^{2}}{4}
$$

С практической точки зрения, регистрируя разность частот или энергий, вылетевших в противоположных направлениях квантов, используя максимальное значение этой разности за время измерения $\Delta \omega_{\max }$, можно 
оценить действительную скорость движения позитронов (21) перед аннигиляцией. Скорость позитрона после его замедления в тканях зависит от механических параметров ткани, через которую он движется: плотности, вязкости и т.д, поэтому в процессе диагностики организма с помощью ПЭТ можно получить дополнительные механические характеристики ткани.

\section{Заключение}

По результатам проведенного анализа можно сделать следующие выводы.

Формулы (1) и (2) адекватно в разных системах отсчета описывают разлет фотонов при аннигиляции электрона и позитрона.

В лабораторной системе отсчета, связанной с электроном (или позитронно-эмиссионным томографом), угловое распределение количества вылетающих фотонов сферически-симметрично, однако за счет различия в условиях наблюдения квантов вследствие эффекта Доплера возникает различие в частотах излученных квантов.

При переходе в систему отсчета, связанную с центром масс электрона и позитрона, различие в частотах излученных квантов редуцируется в угловое распределение интенсивности аннигиляционного излучения, которое также является следствием эффекта Доплера.

Исследуя угловое распределение интенсивности электромагнитного излучения при аннигиляции позитрона и электрона в системе их центра масс, мы исследуем не аннигиляцию, а совершенно другое физическое явление - эффект Доплера, который сопровождает аннигиляционное излучение.

Измеряя разность частот или энергий квантов, вылетающих в противоположных направлениях, можно оценить скорость движения позитронов. Учитывая связь скорости движения позитрона, после его замедления в тканях организма, с механическими характеристиками ткани, через которые он пролетает: плотностью, вязкостью и т.Д., можно получить информацию о величине этих характеристик.

\section{Список литературы}

[1] Волобуев А.Н. Основы медицинской и биологической физики. Самара: Самарский дом печати, 2011. С. 636.

[2] Dirac P.A.M. Direction in physics / Ed. H. Hora, J.R. Shepanski. NY.: John Wiley \& Sons, 1978. 78 p.

[3] Thomson M. Modern Particle Physics. Cambridge: University Printing House, 2013. P. 96-97.

[4] Гайтлер В. Квантовая теория излучения. М.: ИИЛ, 1956. C. $302-304$

[5] Боголюбов Н.Н., Ширков Д.В. Введение в теорию квантовых полей. М.: Наука, 1976. С. 203-205.

[6] Фейнман Р. Квантовая электродинамика. Курс лекций. М.: Книжный дом ЛИБРОКОМ, 2009. С. 135-137.
[7] Берестецкий В.Б., Лифиии Е.М., Питаевский Л.П. Квантовая электродинамика. М.: Наука, 1989. Т. 4. С. 413, 421.

[8] Ландау Л.Д., Лифиии, Е.М. Теория поля. М.: Наука, 1967. C. 156.

[9] Ициксон К., Зюбер Ж.-Б. Квантовая теория поля. М.: Мир, 1984. C. 280.

[10] Бъеркен Джс.Д., Дрелл С.Д. Релятивистская квантовая теория. М.: Наука, 1978. С. 138.

[11] Яворский Б.М., Детлаф А.А. Справочник по физике. М.: Наука, 1990. С. 576. 